\title{
Disrupted Functional and Structural Networks in Cognitively Normal Elderly Subjects with the APOE $\& 4$ Allele
}

\author{
Yaojing Chen ${ }^{1,2}$, Kewei Chen ${ }^{2,3}$, Junying Zhang ${ }^{1,2}, \mathrm{Xin} \mathrm{Li}^{1,2}$, Ni Shu ${ }^{1,2}$, Jun Wang ${ }^{1,2}$, Zhanjun Zhang*,1,2 and \\ Eric M Reiman ${ }^{3}$ \\ 'State Key Laboratory of Cognitive Neuroscience and Learning and IDG/McGovern Institute for Brain Research, Beijing Normal University, \\ Beijing, China; ${ }^{2} B A B R I$ Centre, Beijing Normal University, Beijing, China; ${ }^{3}$ Banner Alzheimer's Institute, Phoenix, AZ, USA
}

\begin{abstract}
As the Apolipoprotein E (APOE) $\varepsilon 4$ allele is a major genetic risk factor for sporadic Alzheimer's disease (AD), which has been suggested as a disconnection syndrome manifested by the disruption of white matter (WM) integrity and functional connectivity (FC), elucidating the subtle brain structural and functional network changes in cognitively normal $\varepsilon 4$ carriers is essential for identifying sensitive neuroimaging based biomarkers and understanding the preclinical AD-related abnormality development. We first constructed functional network on the basis of resting-state functional magnetic resonance imaging and a structural network on the basis of diffusion tensor image. Using global, local and nodal efficiencies of these two networks, we then examined (i) the differences of functional and WM structural network between cognitively normal $\varepsilon 4$ carriers and non-carriers simultaneously, (ii) the sensitivity of these indices as biomarkers, and (iii) their relationship to behavior measurements, as well as to cholesterol level. For $\varepsilon 4$ carriers, we found reduced global efficiency significantly in WM and marginally in FC, regional FC dysfunctions mainly in medial temporal areas, and more widespread for WM network. Importantly, the right parahippocampal gyrus (PHG.R) was the only region with simultaneous functional and structural damage, and the nodal efficiency of PHG.R in WM network mediates the APOE $\varepsilon 4$ effect on memory function. Finally, the cholesterol level correlated with WM network differently than with the functional network in $\varepsilon 4$ carriers. Our results demonstrated $\varepsilon 4$-specific abnormal structural and functional patterns, which may potentially serve as biomarkers for early detection before the onset of the disease.
\end{abstract}

Neuropsychopharmacology (2015) 40, II8I-II9I; doi:10.1038/npp.2014.302; published online 17 December 2014

\section{INTRODUCTION}

The apolipoprotein $\mathrm{E}(A P O E) \varepsilon 4$ allele is a major genetic risk factor for sporadic Alzheimer's disease (AD) accounting for $40-80 \%$ of cases (Farrer et al, 1997). The increased risk for $\mathrm{AD}$ is 2- to 3 -fold in people with one copy of $\varepsilon 4$ allele and $\sim 12$-fold in those with two copies of $\varepsilon 4$ allele when compared with individuals with no copies of $\varepsilon 4$ allele (Verghese et al, 2011). A longitudinal study of cognitively normal individuals demonstrated that age-related memory decline in $\varepsilon 4$ carriers diverges from that of non-carriers before 60 years of age, suggesting that cognitive impairments in $\varepsilon 4$ carriers begin before the appearance of clinical symptoms (Bookheimer and Burggren, 2009). Thus, elucidating the subtle brain structural and functional changes in older individuals who carry the APOE $\& 4$ allele but are still cognitively normal, can provide information as to the

*Correspondence: Dr Z Zhang, State Key Laboratory of Cognitive Neuroscience and Learning, IDG/McGovern Institute for Brain Research, Beijing Normal University, Beijing 100875, China, Tel: +86 105880 2005, Fax: +86 1058802005 ,

E-mail: zhang_rzs@bnu.edu.cn

Received II September 2014; revised 26 October 2014; accepted 5 November 2014; accepted article preview online 18 November 2014 understanding of $\mathrm{AD}$ pathogenesis, the predicted progression of the disease, and an effective therapeutic window for the early preclinical stages of $\mathrm{AD}$.

Previous studies of normal APOE\& 4 carriers assessed changes either in gray or white matter (WM) in focal brain regions and the inter-regional connectivity. AD-related regional brain functional and structural impairments included a decreased volume of the medial temporal lobe (MTL; Geroldi et al, 1999; Hashimoto et al, 2001), lower cerebral metabolic rates (Reiman et al, 1996), greater whole brain atrophy rates (Chen et al, 2007), and disrupted anterior and posterior WM regions (Nierenberg et al, 2005; Persson et al, 2006; Ryan et al, 2011). It was also reported that behavior/performance or cholesterol levels were associated with certain functional (Nichols et al, 2012) and structural (Ryan et al, 2011) brain damage and with glucose hypometabolism in cognitively normal individuals in an APOE $\varepsilon 4$ status dependent fashion(Reiman et al, 2010).

Primarily owing to the previous inter-region connectivity findings, it has been suggested that $\mathrm{AD}$ may be regarded as a disconnection syndrome that is characterized by the disruption of WM integrity (Zhuang et al, 2010) and functional connectivity (FC; Wang et al, 2013), damage of axonal transport, loss of pyramidal neurons, and reduced 
fidelity of communication between brain regions (Bartzokis et al, 2007; Delbeuck et al, 2003).

Despite the knowledge gained from these studies, little is known about WM alterations in relation to the functional network's and their roles, respectively, in the pivotal degenerative processes in cognitively normal APOE $\varepsilon 4$ allele carriers. The majority of AD-related brain structural studies have not specifically assessed WM network integrity in cognitively normal APOE\&4 carriers, except one that examined primarily the age-related reduction of local interconnectivity within the WM structural network in healthy $A P O E$ 84 carriers(Brown et al, 2011). Moreover, most previously published brain network studies focused on either functional or structural network separately. Analysis of both of these networks simultaneously can provide a better understanding of the role of $A P O E$ in the pathogenesis of $\mathrm{AD}$, including brain $\mathrm{WM}$ integrity/architecture and brain functioning related to interconnected cortical gray matter areas. Moreover, the relationship between these two networks and various physiological parameters, such as cholesterol levels and behaviors, is yet to be further explored. Also, the simultaneous inclusion of information from multiple sources such as the subjects' cognitive performance, $A P O E \& 4$ and the brain functional/structural connectivity information enables us to explicate the possible process that underlies the influence of $A P O E$ on observed relationship between brain networks and the memory performance.

In this study, we aimed to simultaneously examine the WM structural network and functional network and the sensitivity of the indices based on each in comparison with behavioral score early before any clinical symptoms. In addition, we examined the association of these indices in pivotal-disrupted brain regions with memory performance, as well as cholesterol levels. Lastly, we investigated the influence of $A P O E \& 4$ on memory performance using the mediation analysis (Hayes, 2013).

\section{MATERIALS AND METHODS}

\section{Participants}

This study included 75 right-handed, native Chinese subjects. All data are from the Beijing Aging Brain Rejuvenation Initiative (BABRI) database. The study was approved by the Ethics Committee and institutional review board of Beijing Normal University Imaging Center for Brain Research, and written informed consent was given by all participants. To be included in this study, participants had to meet the following criteria: (i) a score of at least 24 on the Mini Mental-Status Examination (MMSE); (ii) no history of neurologic, psychiatric, or systemic illnesses known to influence the cerebral function, including serious vascular diseases, head trauma, tumor, current depression, alcoholism, and epilepsy; (iii) no prior history of taking psychoactive medications; (iv) to be able to cope with the physical demands of the MR image; (v) the Clinical Dementia Rating was 0; and (vi) not taking lipid-lowering medication. The following exclusion criteria were used in this study: (a) structural abnormalities other than cerebrovascular lesions, such as tumors, subdural hematomas, and contusions because of previous head trauma, that could impair cognitive function; (b) history of addictions, neurologic or psychiatric diseases, or treatments that would affect cognitive function; (c) large vessel disease, such as cortical or subcortical infarcts and watershed infarcts; and (d) diseases with WM lesions, such as normal-pressure hydrocephalus and multiple sclerosis.

\section{Neuropsychological Testing}

All participants underwent a battery of neuropsychological tests that assessed their general mental status and other cognitive domains, such as memory, attention, spatial processing, executive function and language ability as detailed in Supplementary Materials. Thirteen neurocognitive measures were derived from 10 separate neuropsychological tests (Table 1).

\section{Analysis of Genetic and Biochemical Indicators}

DNA was extracted from the blood samples of subjects according to standard procedures for the subsequent characterization of APOE genotype using PCR (Applied Biosystems, Foster City, CA). All participants were genotyped for two SNPs in the APOE gene (rs429358 and rs7412) using previously published methods (Felsky et al, 2012). There were 35 APOE $\varepsilon 4$ carriers (including $32 \varepsilon 3 / \varepsilon 4$ genotype and $3 \varepsilon 4 / \varepsilon 4$ genotype) and 40 APOE $\varepsilon 4$ non-carriers (including $30 \quad \varepsilon 3 / \varepsilon 3$ genotype and $10 \quad \varepsilon 2 / \varepsilon 3$ genotype) included in our present study. Blood samples were collected at Beifang hospital at the same time of the day. The serum total cholesterol (TC), triglyceride (TG), high-density lipoprotein and low-density lipoprotein (LDL) levels were measured with an Automatic Biochemistry Analyzer (OLYMPUS AU400, Japan) using commercially available diagnostic kits (Beckman Coulter, Ireland).

\section{MRI Data Acquisition}

All participants were scanned with a SIEMENS TRIO 3T scanner in the Imaging Center for Brain Research at Beijing Normal University, including high-resolution T1-weighted structural MRI, diffusion tensor imaging (DTI) and resting-state functional MRI (rsfMRI) scans. The detailed parameters are provided in Supplementary Materials. Participants laid supine with their head fixed snugly by straps and foam pads to minimize head movement.

\section{Data Preprocessing}

DTI data were performed with the FDT toolbox in FSL (www.fmrib.ox.ac.uk/fsl). Functional data were preprocessed using SPM8 (www.fil.ion.ucl.ac.uk/spm/software/spm8/) and DPARSF (www.restfmri.net/orum/PARSF) software. The detailed preprocessing steps are provided in Supplementary Materials.

\section{Brain Network Construction}

The brain network constructions for DTI and rsfMRI data each are based on the approach previously reported (Shu et al, 2012; Zhang et al, 2011) and detailed below. Nodes and edges are the two basic elements of a network. To define the 
Table I Demographic, Neuropsychological and Clinical Characteristics of APOE $\varepsilon 4$ Carriers and Non-Carriers

\begin{tabular}{|c|c|c|c|c|}
\hline & APOE $\& 4$ carriers $(n=35)$ & APOE $\varepsilon 4$ non-carriers $(n=40)$ & F/T-value $\left(\chi^{2}\right)$ & $p$-value \\
\hline Age (years) & $67.26 \pm 7.62$ & $64.45 \pm 7.30$ & 1.63 & $0.11^{a}$ \\
\hline Male/female & $17 / 18$ & $19 / 21$ & 0.01 & $1.00^{\mathrm{b}}$ \\
\hline Education (years) & $|1.3| \pm 3.23$ & $10.75 \pm 3.13$ & 0.77 & $0.44^{\mathrm{a}}$ \\
\hline \multicolumn{5}{|l|}{ General mental status } \\
\hline \multicolumn{5}{|l|}{ Memory function } \\
\hline AVLT-delay recall & $4.23 \pm 2.95$ & $3.68 \pm 2.61$ & 0.42 & 0.52 \\
\hline ROCF-delay recall & $11.11 \pm 6.79$ & $10.53 \pm 6.87$ & 0.01 & 0.92 \\
\hline Backward digit span & $4.45 \pm 1.504$ & $3.87 \pm 1.25$ & 2.54 & 0.12 \\
\hline \multicolumn{5}{|l|}{ Visuo-spatial processing } \\
\hline \multicolumn{5}{|l|}{ Language } \\
\hline CVFT & $43.51 \pm 10.76$ & $40.23 \pm 7.91$ & 1.52 & 0.22 \\
\hline BNT & $23.7 \mid \pm 3.87$ & $23.38 \pm 4.02$ & 0.01 & 0.94 \\
\hline \multicolumn{5}{|l|}{ Attention } \\
\hline SDMT & $31.83 \pm 11.70$ & $33.08 \pm 10.89$ & 0.37 & 0.55 \\
\hline SCWT-B Time & $41.77 \pm 10.27$ & $39.68 \pm 7.01$ & 0.52 & 0.47 \\
\hline TMT-A time (s) & $64.7| \pm 34.7|$ & $55.56 \pm 24.86$ & 1.06 & 0.31 \\
\hline \multicolumn{5}{|l|}{ Lipid indicators } \\
\hline $\mathrm{TG}(\mathrm{mmol} / \mathrm{l})$ & $1.69 \pm 1.01$ & $2.38 \pm 3.49$ & 1.20 & 0.28 \\
\hline $\mathrm{HDL}(\mathrm{mmol} / \mathrm{l})$ & $1.30 \pm 0.32$ & $1.32 \pm 0.31$ & 0.44 & 0.51 \\
\hline LDL (mmol/l) & $2.91 \pm 1.12$ & $3.34 \pm 0.87$ & 2.19 & 0.15 \\
\hline
\end{tabular}

Abbreviations: AVLT, auditory verbal learning test; BNT, Boston naming test; CDT, clock-drawing test; CVFT, category verbal fluency test; HDL, high-density lipoprotein; LDL, low-density lipoprotein; MMSE, Mini Mental-Status examination; ROCF, Rey-Osterrieth complex figure test; SCWT, stroop color and word test; SDMT, symbol digit modalities test; TC, total cholesterol; TG, triglyceride; TMT, trail making test.

Values are mean \pm SD or number of participants. The comparison of neuropsychological scores, glucose, and lipid levels between the two groups was performed with an analysis of covariance.

${ }^{a}$ The $p$-value for age and education were obtained using an independent two-sample $t$-test.

${ }^{\mathrm{b}}$ The $p$-value for sex was obtained using a $\chi^{2}$ test.

brain nodes of WM and functional networks, a prior atlas of automated anatomical labeling was used to divide the whole brain into 90 (45 for each hemisphere) regions (Supplementary Table S1).

WM structural network construction. The nodes were defined in native space for each individual using the procedure proposed by Gong et al (2009). Using this procedure, we obtained 90 nodes for the WM network. Diffusion tensor tractography was implemented with DTI-studio software (H. Jiang, S. Mori, Johns Hopkins University) by using the 'fiber assignment by continuous tracking' method (Mori et al, 1999). Specifically, we defined the average fractional anisotropy (FA) along the pathways of the interconnecting streamlines between two regions as the weight of the network edges. Thus, for each participant we constructed the FA-weighted WM network that was 
represented by a symmetric $90 \times 90$ matrix (Supplementary Materials).

Functional network construction. ROI time series were corrected for patient movement, the global mean signal, the WM signal and the cerebrospinal fluid signal. For measurement of the network edges, we calculated the Pearson's correlation coefficients between corrected time series of every pair of all ROIs. The edge weight for each pair was defined as the correlation coefficient for those who survived a statistical threshold $(p<0.05$, Bonferroni-corrected $)$. Otherwise, the edge weights were assigned as 0 . Thus, we yielded a symmetric $90 \times 90$ weighted FC network matrix that represented the strength of the functional link between any two regions for each individual.

\section{Network Analysis}

To characterize the topological organization of networks, global efficiency, and local efficiency graph measures were assessed (Rubinov and Sporns, 2010). Efficiency is biologically meaningful as it reflects how well the information propagates over a network. For regional characteristics, we analyzed the nodal efficiency (Achard and Bullmore, 2007), which is a centrality measure and has been frequently used and serves as a potential marker to assess the risk of $\mathrm{AD}$ (Lo et al, 2010; Shu et al, 2012). All network analyses were performed using in-house GRETNA software (www.nitrc. org/projects/gretna/) and visualized using BrainNet Viewer software (www.nitrc.org/projects/bnv/; Supplementary Materials).

\section{ROC Analysis}

Receiver Operating Characteristic (ROC) analysis was performed using the MedCalc Package (http://www.medcalc.org/).

\section{Mediation Analysis}

The mediation analysis (Hayes, 2013) was conducted using SPSS software. The significance threshold for the Sobel test was set at $p<0.05$.

\section{Statistical Analysis}

Independent two-sample $t$-tests were used to assess between-group differences in age and education. The $\chi^{2}$ test was used to compare gender ratio difference. For neuropsychological assessment, lipid levels, global network topological (global efficiency and local efficiency), and regional (nodal efficiency) measures, an analysis of covariance was used to test between-group differences (adjusted age, gender, and education).For the multiple nodal efficiencies, we applied the false-discovery rate (FDR) procedure to correct the multiple comparisons at a $q$-value of 0.05 . Pearson's correlation analyses were used separately for the $\varepsilon 4$ carriers and non-carriers to assess the relationships between common altered nodal efficiency of two modality networks and lipid levels without the influence of age, gender, and education.

\section{RESULTS}

\section{Demographic, Neuropsychological, and Lipid Panel Measurements}

Characteristics, neuropsychological test scores and serum lipid panel measurements of two groups are shown in Table 1. No differences in age, gender, or education were found between carriers and non-carriers. After controlling for the effects of age, gender, and education, there were no statistically significant group differences in any neuropsychological measure or lipid levels.

\section{Global Topological Organization of Networks}

Structural and functional networks were constructed individually for each subject. Connections of overall ROI pairs for structural and functional networks were depicted for carriers and non-carriers (Supplementary Figure S1). We found that both carriers and non-carriers showed smallworld organization of functional and structural networks expressed as $\gamma>1$ and $\lambda \sim 1$ (Supplementary Figure S2). Carriers had a significantly lower global efficiency in the structural brain network $(p=0.003$, carriers: $0.73 \pm 0.05$, non-carriers: $0.77 \pm 0.05)$ and marginally lower in functional network $(p=0.054$, carriers: $0.50 \pm 0.02$, non-carriers: $0.52 \pm 0.03)$, with the effects of age, gender, and education as covariates. There were no significant differences in the local efficiency of the structural network $(p>0.1$, carriers: $1.07 \pm 0.04$, non-carriers: $1.09 \pm 0.05$ ) or functional network ( $p>0.1$, carriers: $0.80 \pm 0.04$, non-carriers: $0.82 \pm 0.04$; Figure 1a).

\section{Regional Topological Organization of Networks}

For the between-group nodal efficiency comparisons, covariates of age, gender, and education were applied. The mean nodal efficiency for each group depicted the overall topography of efficiency across the brain functional network and structural network (Figure 1b). In the functional network, a number of temporal regions showed significant decreases in nodal efficiency in carriers with a statistical threshold of $q<0.05$, FDR-corrected, including bilateral hippocampus (HIP), right parahippocampal gyrus (PHG.R), bilateral amygdala (AMYG), and right heschl gyrus (Figure 2a). In the WM network, between-group comparisons revealed that four brain structures $(q<0.05$, FDR-corrected $)$ showed decreased nodal efficiency in carriers, including the left anterior cingulate and paracingulate gyrus (ACG.L), right dorsolateral superior frontal gyrus(SFGdor.R), PHG.R, and left inferior occipital gyrus (IOG.L; Figure $2 \mathrm{~b}$ and Supplementary Table S2).

\section{ROC Analysis}

We used the ROC analysis to determine whether the network measures might have a greater diagnostic ability than the MMSE for APOE $\& 4$ carriers. The network measures include global efficiency (e.g., Global efficiency_FUN and Global efficiency_WM) and mean nodal efficiency of significant decreasing regions (e.g., Decreasing_region_FUN, and Decreasing_region_WM). The area under the ROC curve 
a

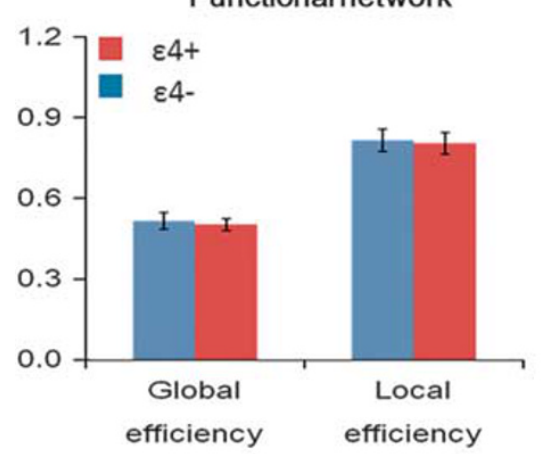

White matter network

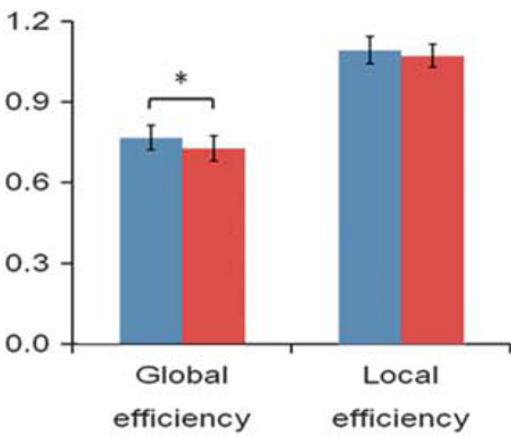

b

Functional network

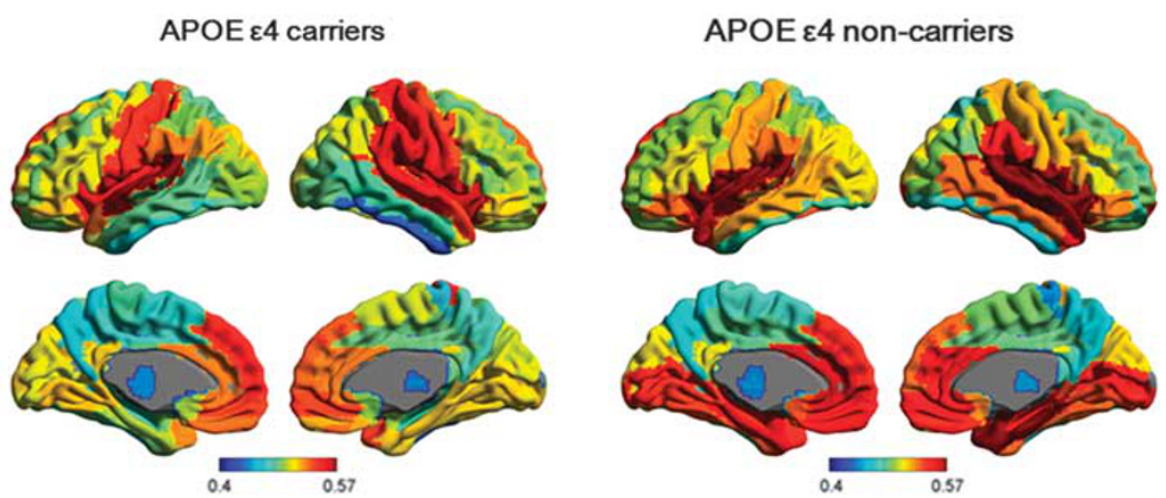

White matter network
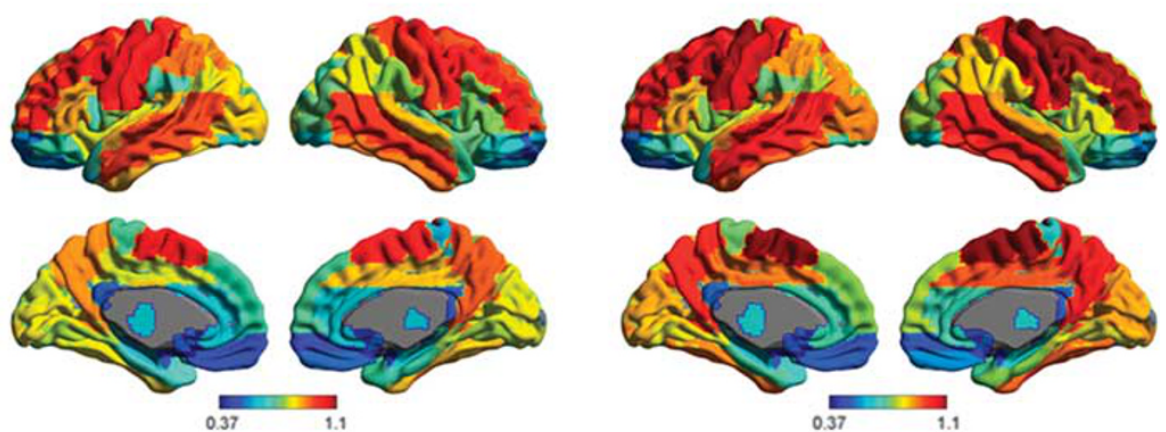

Figure I (a) Group differences in global measures of functional and structural networks were quantified for APOE $\varepsilon 4$ carriers and non-carriers. Differences between groups were assessed using an analysis of covariance adjusted for age, sex, and education. Error bars represent the SD. (b) The mean nodal efficiency of the functional network and structural network in carriers and non-carriers.

\section{Functional network}
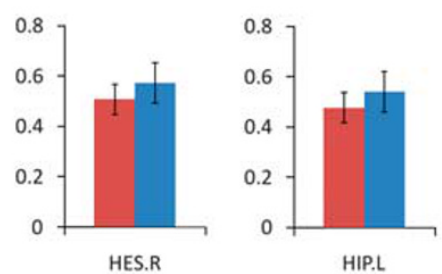

HIP.L

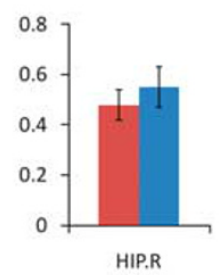

White matter network
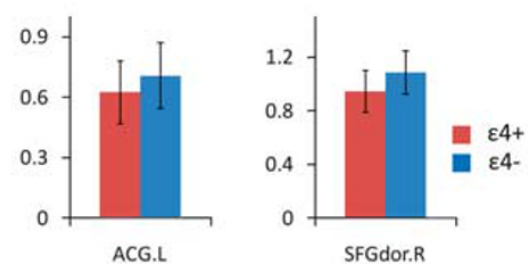

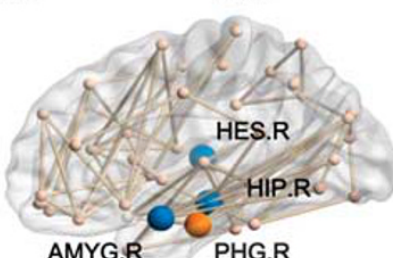

AMYG.R PHG.R
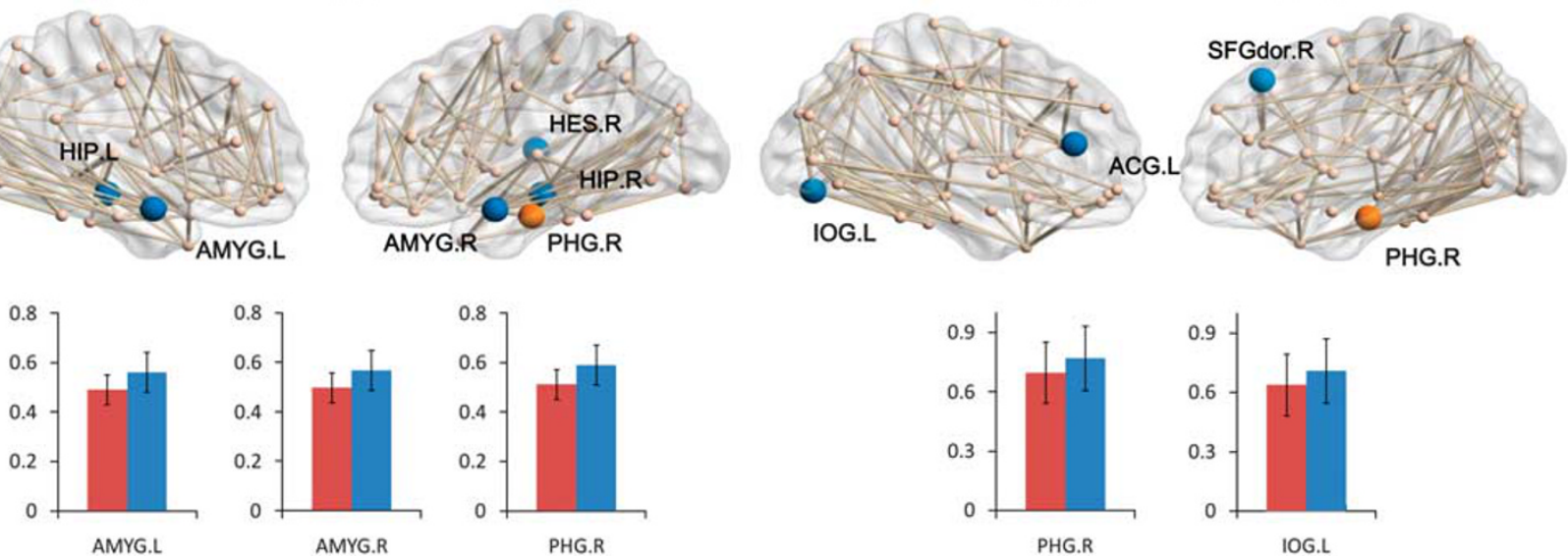

Figure 2 Brain regions with significant group differences in nodal efficiency between carriers and non-carriers. All regions colored in red and blue have a significantly lower nodal efficiency for carriers $(q<0.05$, FDR-corrected). The PHG.R colored in red indicates the common disrupted region in structural and functional networks. Error bars represent the SD. 
(AUC) of MMSE (AUC $=0.51$ ) was significantly smaller than that WM network indices (Global efficiency_WM:0.74, Decreasing_region_WM:0.81) and functional networks indices (Global efficiency_FUN:0.70, Decreasing_region_ FUN:0.79). The mean nodal efficiency yielded greater diagnostic accuracy than global efficiency in any network (Supplementary Figure S3, Supplementary Table S3, and Supplementary Table S4).

\section{Mediation Analysis}

To address the question of whether common altered regional efficiencies of the two networks mediated the association between the $A P O E$ genotype and the memory measures, the mediation analysis was applied. The independent factor was $A P O E$ genotype and dependent variables were memory measures, such as AVLT-delay recall, ROCFdelay recall or Backward digit span. The proposed mediator was the nodal efficiency of PHG.R in the WM and functional network separately, which showed significant genotype effects in both networks. As shown in Figure 3a, mediation analysis indicated that the nodal efficiency of PHG.R in WM network mediates the effect of $A P O E$ variants on ROCF-delay recall performance $(z=-2.33, p=0.02)$. There was a significant association between PHG.R efficiency and $A P O E$ genotype, as well as between PHG.R efficiency and ROCF-delay recall performance, but no significant association between APOE genotype and ROCF-delay recall performance.

Considering the potential differences caused by cognitive status, we divided the $\varepsilon 4$ carriers into two subgroups according to the ROCF-delay recall score (the higher memory group equaled the top $50 \%$ and the lower memory group equaled lower $50 \%$ ). We found significant correlations in the lower memory performance group $(r=0.71$, $p=0.001$; Figure $3 \mathrm{~b}$ ). Further mediation analysis showed a significant relationship between APOE genotype and ROCFdelayed recall performance and a significant mediating effect through PHG.R efficiency in the lower-scoring subgroup, but the higher-scoring subgroup yielded no significant results (Supplementary Figure S4A).

No significant mediation effect of PHG.R efficiency was found for the AVLT-delayed recall and Backward digit span performances (Supplementary Figure S4B, and C).

\section{Nodal Efficiency of PHG.R Correlates with Lipid Levels}

Finally, the relationship between PHG.R efficiency in each $\mathrm{WM}$ and functional networks and cholesterol levels were tested, respectively, for $A P O E \& 4$ carriers and non-carriers, after controlling for the effects of age, gender, and education. PHG.R efficiency of the WM network was positively correlated with TC only in non-carriers $(r=0.43, p=0.015)$ not in carriers $(r=-0.05, p=0.83)$, and similarly with LDL levels (carriers: $r=0.21, p=0.37$, non-carriers: $r=0.42$, $p=0.02$; Figure 4 , left column). Interestingly, there was a significant negative relationship between PHG.R efficiency of functional network and TC in non-carriers $(r=-0.42$, $p=0.018))$ not carriers $(r=-0.09, p=0.71)$ and similarly for LDL levels (carriers: $r=-0.08, p=0.73$, non-carriers: $r=-0.46, p=0.01$; Figure 4 , right column). Notably, the significant correlation results were not corrected for multiple comparisons and therefore should be regarded as exploratory in nature.

\section{DISCUSSION}

Our study evaluated the differences in large-scale brain functional networks and WM structural topologies between $A P O E \& 4$ carriers and non-carriers. The results showed that $A P O E \& 4$ carriers, although cognitively normal, already exhibited aberrant $\mathrm{AD}$-related functional and especially structural organization changes on global efficiency. At the local level, the functional network in $\varepsilon 4$ carriers had a decreased nodal efficiency in MTL areas, whereas their a

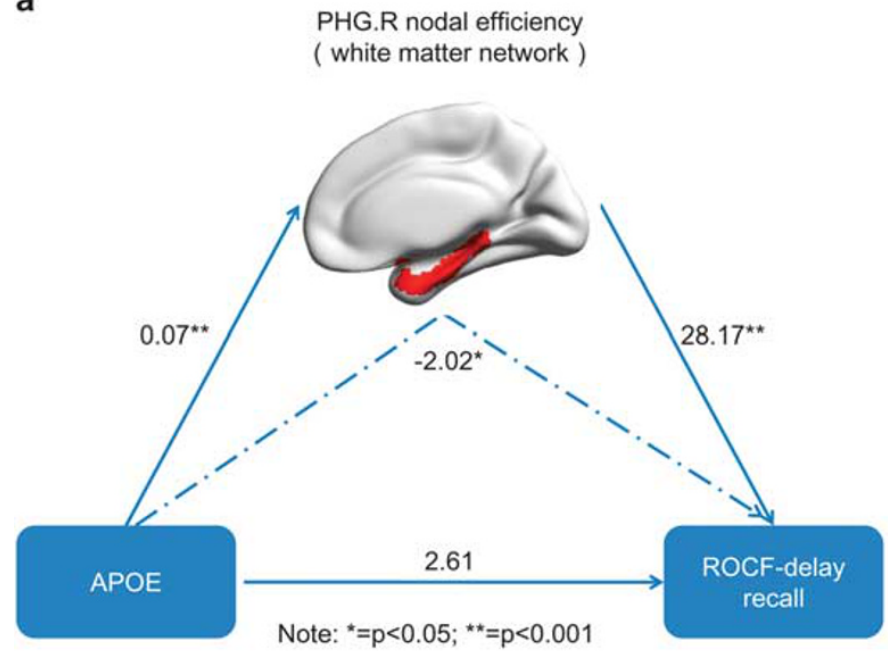

b

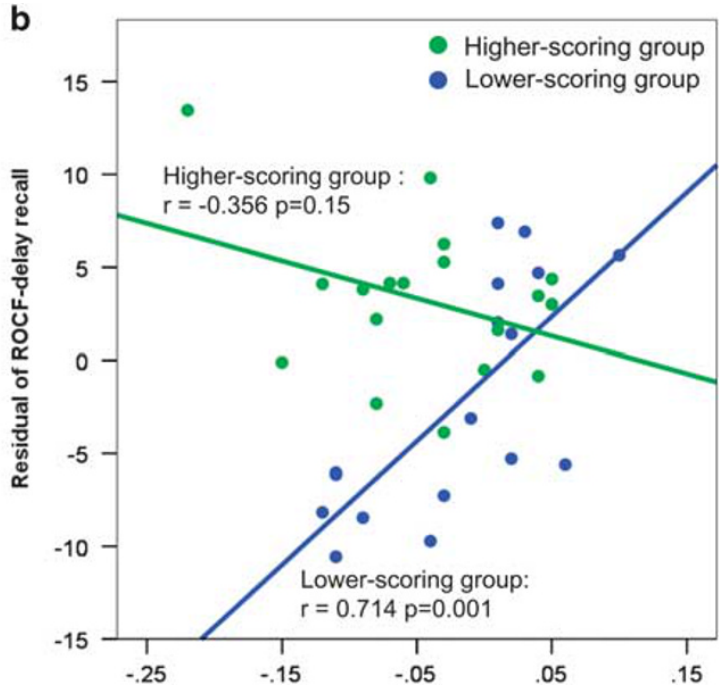

Figure 3 (a) Mediation model illustrating the direct effect of APOE genotype on PHG.R efficiency of the WM network, the direct effect of APOE genotype on ROCF-delay recall performance, the direct effect of PHG.R efficiency on ROCF-delay recall performance and the mediating effect of PHG.R efficiency on the association between APOE genotype and ROCF-delay recall performance. As indicated by the path coefficients and the $p$-values. (b) Scatterplots of the association between PHG.R efficiency and ROCF-delay recall in lower-scoring and higher-scoring $\varepsilon 4$ carrier subgroups. 

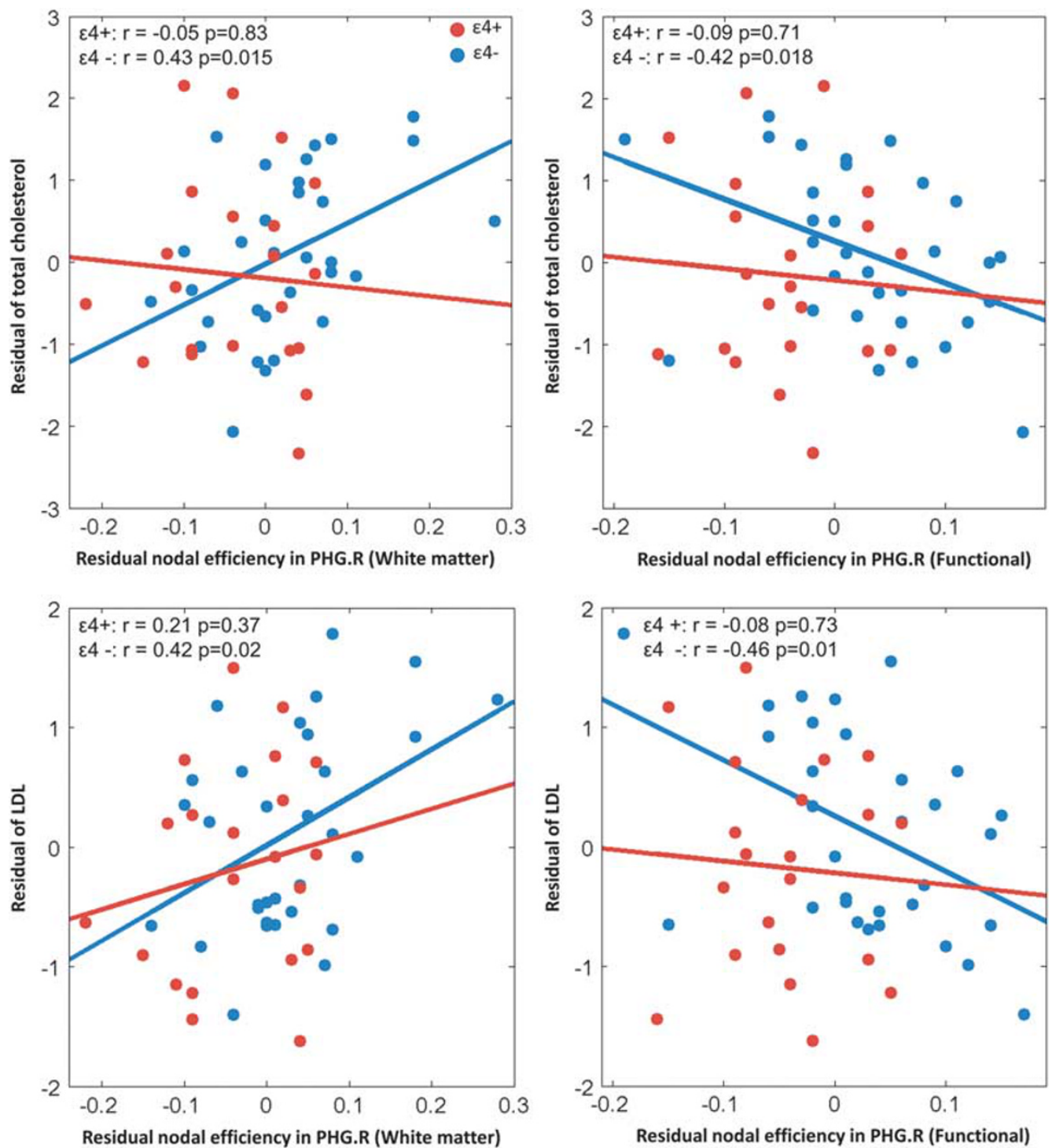

Figure 4 The correlation of PHG.R regional efficiency and cholesterol levels in APOE $\varepsilon 4$ carriers and non-carriers.

structural network had aberrant regional topological patterns that extended beyond the temporal lobe to additional regions, such as ACC.L, SFG.R and IOG.L. Moreover, both the functional and structural regional efficiencies were both decreased particularly in the right PHG region in 84 carriers. Extensive analysis revealed that this specific region, as part of the WM network, mediates the APOE effect on memory function. Finally, we found that that the cholesterol level correlated with the WM network differently compared with the functional network in $A P O E \& 4$ carriers.

\section{Disrupted Organization of the WM Structural and Functional Networks}

The brain is a complex system of interconnected regions. One major finding of our study is that the global properties of both the WM and functional networks were reduced in APOE \&4 carriers. Similarly, reduced global connectedness in patients with $\mathrm{AD}$ was also reported for structural (Lo et al, 2010) and functional networks(Stam et al, 2007). Thus, the APOE $\& 4$ allele may manifest functional and WM brain network abnormalities before the expression of any clinical or neurophysiological impairment.

In addition to the observed global properties, APOE $\varepsilon 4$ related regional WM network changes were identified in the SFGdor.R, ACG.L, PHG.R, and IOG.L. The PHG has been strongly implicated in AD pathophysiology (Van Hoesen et al, 2000) and associated with the APOE 84 allele (Honea et al, 2009; Nierenberg et al, 2005). Post-mortem studies have demonstrated a severe neuronal loss in the entorhinal cortex (PHG contains most of the entorhinal cortex) even in very mild $\mathrm{AD}$ (Hyman et al, 1984) and mild cognitive impairments (MCI) (Kordower et al, 2001). Furthermore, reduced WM nodal efficiencies of SFGdor and ACG in AD (Lo et al, 2010) and MCI (Shu et al, 2012) have been reported. A recent study suggested that the APOE $\varepsilon 4$ genotype may participate in brain-derived neurotrophic factor (BDNF) metabolism (Liu et al, 2014), which is necessary 
and sufficient to induce long-lasting structural changes in dendritic spines and that their enlargement could be blocked by inhibiting protein synthesis (Tanaka et al, 2008). Notably, the regions we reported in this study represent earlier pathological events and go beyond the traditional core regions of default mode network that were found to have significant age-related reductions (Brown et al, 2011).

In the functional network, decreased nodal efficiency was mainly found in MTL regions (e.g., HIP, AMYG, and PHG) and in the HES, which is consistent with the previous studies (Machulda et al, 2011; Sheline et al, 2010). Importantly, a MTL connectivity disturbance was also shown in amnestic type MCI (Bai et al, 2009) and AD patients (Zhou et al, 2008). This result highlights the differences between the WM and functional networks, indicating that dysfunctions in the WM network are more widespread, whereas the functional dysfunctions mainly initiate from memory-associated MTL regions.

In addition to the network differences related to $\varepsilon 4$ status at the group level, the ROC analysis showed that the AUCs were significantly larger for network measures than the MMSE. Our study directly supports the notion that the pathological changes in the brain of cognitively normal people with genetic risks of dementia begin to emerge many years or decades before any clinical symptoms appear (Bateman et al, 2012; Fleisher et al, 2012).

\section{The PHG Region and the Lateralization}

To our knowledge, our study is the first to report both functional and structural changes of PHG.R nodal efficiency deficits in $A P O E \& 4$ carriers. Structurally, previous studies revealed that $\varepsilon 4$ may influence the integrity of PHG WM in the ways of a decrease in WM volume and an increase in mean diffusivity (Nierenberg et al, 2005; Wang et al, 2012), and ultimately degrade sensory information processing in HIP owing to the disrupted afferent connection from the PHG (Van Hoesen et al, 1991). Moreover, 84 carriers showed a significantly increased number of senile plaques in the PHG when compared with the non-carriers (Sparks, 1997). These findings suggests that $A P O E$ status might play a key role in the maintenance and regeneration of synapses in the PHG (Masliah et al, 1996).Functionally, one investigation showed significant declines in the cerebral metabolic rate for glucose in the PHG in cognitively normal $\varepsilon 4$ heterozygotes (Reiman et al, 2001). Consistent with the aberrant MTL connectivity described above, cognitively normal $\varepsilon 4$ elderly subjects were found to have a disruption in PHG connectivity (Sheline et al, 2010).Moreover, the majority of fMRI studies in $\varepsilon 4$ carriers found an additive effect of the $\varepsilon 4$ allele on the level of blood-oxygen-level dependent activity in the PHG during episodic memory tasks, which is generally considered as the most vulnerable cognitive function in AD pathology. Specifically, these concurrent functional and structural changes in the right PHG might be a consequence of the early events of $A P O E$-mediated degeneration of neurons and disruption of connections.

With regard to laterality, our post hoc analysis found that the PHG.L exhibited a generally lower efficiency than the PHG.R in both networks, either in $\varepsilon 4$ carriers or noncarriers (Supplementary Figure S5). In general, there was a greater age-related effect on brain structure in the left hemisphere (Davatzikos and Resnick, 2002), particularly in the temporal lobe (Resnick et al, 2003). Existing studies have also suggested that $A P O E \& 4$ carriers with $\mathrm{AD}$, as well as older cognitively healthy individuals showed thinner entorhinal cortex in the left hemisphere when compared with the right hemisphere (Donix et al, 2013).The disrupted nodal efficiency in the PHG.R in both the functional and WM network may suggest that $A P O E \& 4$ carriers could develop a more severe $\mathrm{AD}$-related destruction than noncarriers in the PHG.R.

\section{The PHG.R, Memory Function and APOE 84}

It is important to integrate knowledge of genes, the brain, and behavior to further assess the $A P O E$-related abnormalities of both structural and functional networks, especially with regard to the PHG.R. Using a mediation analysis, we found that differences between $\varepsilon 4$ carriers and non-carriers in memory performance (ROCF-delay recall) were mediated by the PHG.R efficiency in the WM network, particularly in the lower-scoring subgroup. As a pivotal position for mediating memory functions, the PHR receives inputs from widespread secondary cortical regions and provides the major conduit for hippocampal outputs to the same cortical association areas. In line with this reasoning and our mediation analysis result, an MRI report found that PHG volume, specifically in the right hemisphere, was positively related to delayed recall of memory tasks (Kohler et al, 1998). Furthermore, a study in the healthy aging population found significantly more PHG atrophy in the group with memory decline when compared with the group with a stable memory (Burgmans et al, 2011). Some neuropsychological findings indicated the critical role of the PHG in recognition memory, independent from the intermediary of cortical-hippocampal interactions (Eichenbaum et al, 1994; Murray, 1996). Thus, $\varepsilon 4$ seems to have a modifying effect on the baseline cognition associated with PHG structure and memory in early stage. Overall, $\varepsilon 4$ carriers whom exhibit memory decline are at high risk for developing AD (Dik et al, 2000) and have a greater impairment in brain structures.

\section{The PHG.R, Lipid Levels and APOE 84}

As a major lipid transporter and redistribution regulator in the brain (Mahley et al, 2006), impaired apoE function exhibited by $A P O E-\varepsilon 4$ carriers may give rise to abnormal cholesterol homeostasis (Jiang et al, 2008; Riddell et al, 2008). Considering that cholesterol has a critical role in WM integrity, including membrane repair and maintenance of the myelin sheath (Ignatius et al, 1987). Furthermore, $\varepsilon 4$ was reported to be associated with the inhibition of neurite extension (Masliah et al, 1995) and the deficient capacity of cholesterol delivery (Hamanaka et al, 2000). Accordingly, our findings support that higher lipid levels may have a vital role to assure better WM integrity with aging resulting in more effective WM efficiency in non-carriers. Conversely, for functional PHG.R efficiency, we found that lower lipids levels were associated with higher functional efficiency. It is important to note that the serum cholesterol, specifically LDL, may circulate in the brain and is closely related to 
cerebral cholesterol (Dehouck et al, 1997). In addition, $\varepsilon 4$ is associated with a deficient capacity for cholesterol delivery (Rapp et al, 2006) and weak interactions with blood lipids that gives rise to abnormal CNS cholesterol homeostasis. Given that normal cortical function relies highly on regional metabolism, our results coincide with the study reporting an association of lower TC levels with higher glucose metabolism (indicating a reduction in neuronal activity; Reiman et al, 2010).

\section{Limitations}

It was reported that the $\varepsilon 4$ allele has a dose effect on $\mathrm{AD}$ risk (Corder et al, 1993). With only three $\varepsilon 4$ homozygotes, we were not able to assess this dose effect. Next, assessment of tractography quality is dependent on personal experience, and so certain subjective judgments exist. Therefore, more quantitative validation shall be performed in future studies.

\section{CONCLUSION}

Our results indicate that cognitively normal elderly $\varepsilon 4$ carriers already exhibit topological alterations in both WM and functional networks. This was characterized by more widespread network dysfunction in the WM network and dysfunction located mainly in MTL areas for the functional network. It is worthwhile to note both networks show decreased efficiency in the PHG.R, an area that mediates the effect of $A P O E$ variants on memory function and correlates with cholesterol level.

\section{FUNDING AND DISCLOSURE}

Financial support for this study was provided by Beijing New Medical Discipline Based Group 100270569 (ZZ); by Natural Science Foundation of China 30873458 and 81173460 (ZZ); by State Key Program of National Natural Science of China 81430100 (ZZ); by project of Institute of Basic Research in Clinical Medicine, China Academy of Chinese Medical Sciences Z0175 (ZZ); by program for New Century Excellent Talents in UniversityNCET-10-0249 (ZZ); by National Institute on Aging R01AG031581 and P30AG19610 (EMR). The authors declare no conflict of interest.

\section{ACKNOWLEDGEMENTS}

ZZ had full access to all of the data in the study and takes responsibility for the integrity of the data and the accuracy of the data analysis. ZZ and EMR conceived the original idea for the study, supervised in the conception and revised the manuscript. YC, JZ, XL, NS, and JW recruited the study population and conducted the neuropsychological tests. YC and KC analyzed the data. YC, KC, and ZZ drafted the manuscript. All authors read and approved the final manuscript.

\section{REFERENCES}

Achard S, Bullmore E (2007). Efficiency and cost of economical brain functional networks. PLoS Comput Biol 3: e17.
Bai F, Watson DR, Yu H, Shi Y, Yuan Y, Zhang Z (2009). Abnormal resting-state functional connectivity of posterior cingulate cortex in amnestic type mild cognitive impairment. Brain Res 1302: 167-174.

Bartzokis G, Lu PH, Mintz J (2007). Human brain myelination and amyloid beta deposition in Alzheimer's disease. Alzheimers Dement 3: 122-125.

Bateman RJ, Xiong C, Benzinger TL, Fagan AM, Goate A, Fox NC et al (2012). Clinical and biomarker changes in dominantly inherited Alzheimer's disease. N Engl J Med 367: 795-804.

Bookheimer S, Burggren A (2009). APOE-4 genotype and neurophysiological vulnerability to Alzheimer's and cognitive aging. Annu Rev Clin Psychol 5: 343-362.

Brown JA, Terashima KH, Burggren AC, Ercoli LM, Miller KJ, Small GW et al (2011). Brain network local interconnectivity loss in aging APOE-4 allele carriers. Proc Natl Acad Sci USA 108: 20760-20765.

Burgmans S, van Boxtel M, van den Berg K, Gronenschild E, Jacobs H, Jolles J et al (2011). The posterior parahippocampal gyrus is preferentially affected in age-related memory decline. Neurobiol Aging 32: 1572-1578.

Chen K, Reiman E, Alexander G, Caselli R, Gerkin R, Bandy D et al (2007). Correlations between apolipoprotein E $\varepsilon 4$ gene dose and whole brain atrophy rates. Am J Psych 164: 916-921.

Corder EH, Saunders AM, Strittmatter WJ, Schmechel DE, Gaskell PC, Small GW et al (1993). Gene dose of apolipoprotein E type 4 allele and the risk of Alzheimer's disease in late onset families. Science 261: 921-923.

Davatzikos C, Resnick SM (2002). Degenerative age changes in white matter connectivity visualized in vivo using magnetic resonance imaging. Cereb Cortex 12: 767-771.

Dehouck B, Fenart L, Dehouck MP, Pierce A, Torpier G, Cecchelli R (1997). A new function for the LDL receptor: transcytosis of LDL across the blood-brain barrier. J Cell Biol 138: 877-889.

Delbeuck X, Van der Linden M, Collette F (2003). Alzheimer' Disease as a disconnection syndrome? Neuropsychol Rev 13: 79-92.

Dik MG, Jonker C, Bouter LM, Geerlings MI, van Kamp GJ, Deeg DJ (2000). APOE-epsilon4 is associated with memory decline in cognitively impaired elderly. Neurology 54: 1492-1497.

Donix M, Burggren AC, Scharf M, Marschner K, Suthana NA, Siddarth P et al (2013). APOE associated hemispheric asymmetry of entorhinal cortical thickness in aging and Alzheimer's disease. Psych Res 214: 212-220.

Eichenbaum H, Otto T, Cohen NJ (1994). Two functional components of the hippocampal memory system. Behav Brain Sci 17: 449-471.

Farrer LA, Cupples LA, Haines JL, Hyman B, Kukull WA, Mayeux R et al (1997). Effects of age, sex, and ethnicity on the association between apolipoprotein E genotype and Alzheimer disease. A meta-analysis. APOE and Alzheimer Disease Meta Analysis Consortium. JAMA 278: 1349-1356.

Felsky D, Voineskos AN, Lerch JP, Nazeri A, Shaikh SA, Rajji TK et al (2012). Myelin-associated glycoprotein gene and brain morphometry in schizophrenia. Front Psych 3: 40.

Fleisher AS, Chen K, Quiroz YT, Jakimovich LJ, Gomez MG, Langois CM et al (2012). Florbetapir PET analysis of amyloidbeta deposition in the presenilin 1 E280A autosomal dominant Alzheimer's disease kindred: a cross-sectional study. Lancet Neurol 11: 1057-1065.

Geroldi C, Pihlajamäki M, Laakso M, DeCarli C, Beltramello A, Bianchetti A et al (1999). APOE- $\varepsilon 4$ is associated with less frontal and more medial temporal lobe atrophy in AD. Neurology 53: $1825-1825$.

Gong G, He Y, Concha L, Lebel C, Gross DW, Evans AC et al (2009). Mapping anatomical connectivity patterns of human cerebral cortex using in vivo diffusion tensor imaging tractography. Cereb Cortex 19: 524-536. 
Hamanaka H, Katoh-Fukui Y, Suzuki K, Kobayashi M, Suzuki R, Motegi $\mathrm{Y}$ et al (2000). Altered cholesterol metabolism in human apolipoprotein E4 knock-in mice. Human Mol Genet 9: 353-361.

Hashimoto M, Yasuda M, Tanimukai S, Matsui M, Hirono N, Kazui H et al (2001). Apolipoprotein E $\varepsilon 4$ and the pattern of regional brain atrophy in Alzheimer's disease. Neurology 57: 1461-1466.

Hayes AF (2013). Introduction to mediation, moderation, and conditional process analysis: A regression-based approach. Guilford Press.

Honea RA, Vidoni E, Harsha A, Burns JM (2009). Impact of APOE on the healthy aging brain: a voxel-based MRI and DTI study. J Alzheimer's Dis 18: 553-564.

Hyman BT, Van Hoesen GW, Damasio AR, Barnes CL (1984). Alzheimer's disease: cell-specific pathology isolates the hippocampal formation. Science 225: 1168-1170.

Ignatius MJ, Shooter EM, Pitas RE, Mahley RW (1987). Lipoprotein uptake by neuronal growth cones in vitro. Science 236: 959-962.

Jiang Q, Lee CY, Mandrekar S, Wilkinson B, Cramer P, Zelcer N et al (2008). ApoE promotes the proteolytic degradation of Abeta. Neuron 58: 681-693.

Kohler S, Black SE, Sinden M, Szekely C, Kidron D, Parker JL et al (1998). Memory impairments associated with hippocampal versus parahippocampal-gyrus atrophy: an MR volumetry study in Alzheimer's disease. Neuropsychologia 36: 901-914.

Kordower JH, Chu Y, Stebbins GT, DeKosky ST, Cochran EJ, Bennett D et al (2001). Loss and atrophy of layer II entorhinal cortex neurons in elderly people with mild cognitive impairment. Annals Neurol 49: 202-213.

Liu Y-H, Jiao S-S, Wang Y-R, Bu X-L, Yao X-Q, Xiang Y et al (2014). Associations between ApoE\&4 carrier status and serum BDNF levels-new insights into the molecular mechanism of ApoE\&4 actions in Alzheimer's disease. Mol Neurobiol 1-7.

Lo CY, Wang PN, Chou KH, Wang J, He Y, Lin CP (2010). Diffusion tensor tractography reveals abnormal topological organization in structural cortical networks in Alzheimer's disease. J Neurosci 30: 16876-16885.

Machulda MM, Jones DT, Vemuri P, McDade E, Avula R, Przybelski S et al (2011). Effect of APOE epsilon4 status on intrinsic network connectivity in cognitively normal elderly subjects. Arch Neurol 68: 1131-1136.

Mahley RW, Weisgraber KH, Huang Y (2006). Apolipoprotein E4: a causative factor and therapeutic target in neuropathology, including Alzheimer's disease. Proc Natl Acad Sci USA 103: 5644-5651.

Masliah E, Mallory M, Alford M, Veinbergs I, Roses A (1996). Apolipoprotein $\mathrm{E}$ role in maintaining the integrity of the aging central nervous system. Apolipoprotein $E$ and Alzheimer's Disease. Springer: Berlin Heidelberg, pp 59-73.

Masliah E, Mallory M, Ge N, Alford M, Veinbergs I, Roses AD (1995). Neurodegeneration in the central nervous system of apoE-deficient mice. Exp Neurol 136: 107-122.

Mori S, Crain BJ, Chacko VP, van Zijl PC (1999). Threedimensional tracking of axonal projections in the brain by magnetic resonance imaging. Ann Neurol 45: 265-269.

Murray EA (1996). What have ablation studies told us about the neural substrates of stimulus memory? Semin Neurosci 8: 13-22.

Nichols LM, Masdeu JC, Mattay VS, Kohn P, Emery M, Sambataro F et al (2012). Interactive effect of apolipoprotein e genotype and age on hippocampal activation during memory processing in healthy adults. Arch Gen Psych 69: 804-813.

Nierenberg J, Pomara N, Hoptman MJ, Sidtis JJ, Ardekani BA, Lim KO (2005). Abnormal white matter integrity in healthy apolipoprotein E epsilon4 carriers. Neuroreport 16: 1369-1372.

Persson J, Lind J, Larsson A, Ingvar M, Cruts M, Van Broeckhoven C et al (2006). Altered brain white matter integrity in healthy carriers of the APOE epsilon4 allele: a risk for AD? Neurology 66: 1029-1033.

Rapp A, Gmeiner B, Huttinger M (2006). Implication of apoE isoforms in cholesterol metabolism by primary rat hippocampal neurons and astrocytes. Biochimie 88: 473-483.

Reiman EM, Caselli RJ, Chen K, Alexander GE, Bandy D, Frost J (2001). Declining brain activity in cognitively normal apolipoprotein E epsilon 4 heterozygotes: a foundation for using positron emission tomography to efficiently test treatments to prevent Alzheimer's disease. Proc Natl Acad Sci USA 98: 3334-3339.

Reiman EM, Caselli RJ, Yun LS, Chen K, Bandy D, Minoshima S et al (1996). Preclinical evidence of Alzheimer's disease in persons homozygous for the epsilon 4 allele for apolipoprotein E. $N$ Engl J Med 334: 752-758.

Reiman EM, Chen K, Langbaum JB, Lee W, Reschke C, Bandy D et al (2010). Higher serum total cholesterol levels in late middle age are associated with glucose hypometabolism in brain regions affected by Alzheimer's disease and normal aging. NeuroImage 49: 169-176.

Resnick SM, Pham DL, Kraut MA, Zonderman AB, Davatzikos C (2003). Longitudinal magnetic resonance imaging studies of older adults: a shrinking brain. J Neurosci 23: 3295-3301.

Riddell DR, Zhou H, Atchison K, Warwick HK, Atkinson PJ, Jefferson $\mathrm{J}$ et al (2008). Impact of apolipoprotein E (ApoE) polymorphism on brain ApoE levels. J Neurosci 28: 11445-11453.

Rubinov M, Sporns O (2010). Complex network measures of brain connectivity: uses and interpretations. NeuroImage 52: 1059-1069.

Ryan L, Walther K, Bendlin BB, Lue LF, Walker DG, Glisky EL (2011). Age-related differences in white matter integrity and cognitive function are related to APOE status. NeuroImage 54: 1565-1577.

Sheline YI, Morris JC, Snyder AZ, Price JL, Yan Z, D'Angelo G et al (2010). APOE4 allele disrupts resting state fMRI connectivity in the absence of amyloid plaques or decreased CSF Abeta42. J Neurosci 30: 17035-17040.

Shu N, Liang Y, Li H, Zhang J, Li X, Wang L et al (2012). Disrupted topological organization in white matter structural networks in amnestic mild cognitive impairment: relationship to subtype. Radiology 265: 518-527.

Sparks DL (1997). Coronary artery disease, hypertension, ApoE, and cholesterol: a link to Alzheimer's disease? Annals NY Acad Sci 826: 128-146.

Stam C, Jones B, Nolte G, Breakspear M, Scheltens P (2007). Smallworld networks and functional connectivity in Alzheimer's disease. Cereb Cortex 17: 92-99.

Tanaka J, Horiike Y, Matsuzaki M, Miyazaki T, Ellis-Davies GC, Kasai H (2008). Protein synthesis and neurotrophin-dependent structural plasticity of single dendritic spines. Science 319: 1683-1687.

Van Hoesen GW, Augustinack JC, Dierking J, Redman SJ, Thangavel R (2000). The parahippocampal gyrus in Alzheimer's disease. Clinical and preclinical neuroanatomical correlates. Annals NY Acad Sci 911: 254-274.

Van Hoesen GW, Hyman BT, Damasio AR (1991). Entorhinal cortex pathology in Alzheimer's disease. Hippocampus 1: 1-8.

Verghese PB, Castellano JM, Holtzman DM (2011). Apolipoprotein $\mathrm{E}$ in Alzheimer's disease and other neurological disorders. Lancet Neurol 10: 241-252.

Wang C, Stebbins GT, Medina DA, Shah RC, Bammer R, Moseley ME et al (2012). Atrophy and dysfunction of parahippocampal white matter in mild Alzheimer's disease. Neurobiol Aging 33: 43-52.

Wang J, Zuo X, Dai Z, Xia M, Zhao Z, Zhao X et al (2013). Disrupted functional brain connectome in individuals at risk for Alzheimer's disease. Biol Psych 73: 472-481. 
Zhang J, Wang J, Wu Q, Kuang W, Huang X, He Y et al (2011). Disrupted brain connectivity networks in drug-naive, firstepisode major depressive disorder. Biol Psych 70: 334-342.

Zhou Y, Dougherty JH Jr., Hubner KF, Bai B, Cannon RL, Hutson RK (2008). Abnormal connectivity in the posterior cingulate and hippocampus in early Alzheimer's disease and mild cognitive impairment. Alzheimers Dement 4: 265-270.

Zhuang L, Wen W, Zhu W, Trollor J, Kochan N, Crawford J et al (2010). White matter integrity in mild cognitive impairment: a tract-based spatial statistics study. NeuroImage 53: 16-25.

Supplementary Information accompanies the paper on the Neuropsychopharmacology website (http://www.nature.com/npp) 